\title{
Miniaturization effect of electroosmotic self-propulsive microswimmer powered by biofuel cell
}

\author{
Toshiro Yamanaka (i) and Fumihito Arai
}

\begin{abstract}
For future medical microrobotics, we have proposed the concept of the electroosmotic self-propulsive microswimmer powered by biofuel cell. According to the derived theoretical model, its self-propulsion velocity is inversely proportional to the length of the microswimmer, while it is proportional to the open circuit potential generated by the biofuel cell which does not depend on its size. Therefore, under conditions where those mechanisms work, it can be expected that the smaller its microswimmer size, the faster its self-propulsion velocity. Because of its remarkable feature, this concept is considered to be suitable as propulsion mechanisms for future medical microrobots to move inside the human body through the vascular system, including capillaries. We have already proved the mechanisms by observing the several $10 \mu \mathrm{m} / \mathrm{s}$ velocity of $100 \mu \mathrm{m}$ prototypes fabricated by the optical photolithography using several photomasks and alignment steps. However, the standard photolithography was not suitable for further miniaturization of prototypes due to its insufficient resolution. In this research, we adopted femtosecond-laser 3D microlithography for multi-materials composing of the conductive polymer composites and nonconductive polymer composite and succeeded in fabricating $10 \mu \mathrm{m}$ prototypes. Then we demonstrated more than $100 \mu \mathrm{m} / \mathrm{s}$ velocity of the prototype experimentally and proved its validity of the smaller and faster feature.
\end{abstract}

Keywords: Micro/nano robots, Microswimmer, Glucose biofuel cell, Electroosmotic flow, Conductive/nonconductive polymer composites, Two photon polymerization

\footnotetext{
*Correspondence: yamanaka@biorobotics.mech.nagoya-u.ac.jp Department of Micro/Nano-Mechanical Science and Engineering, Nagoya University, Furo-cho, Chikusa-ku, Nagoya 464-0814, Japan
}

\section{Introduction}

Biomedical swimming microrobots have great potential for use in innovative medicine, such as long-term drug delivery and painless pinpoint surgery. It has been discussed that microrobots autonomously explore the human body through the vascular system and carry out various medical tasks [1]. To achieve this, propulsion and energy supply in microscale biological fluids are the most significant challenges. These developments will require utilizing what can be supplied in the human body as energy sources and applying a propulsion principle that can operate effectively at the microscale. Furthermore,
\end{abstract}

robots capable of swimming against the flow in capillaries are desirable because they can approach most tiny spaces in the body and perform various tasks such as attacking tumors. More specifically, we require mechanisms to realize robots with their size less than a few $10 \mu \mathrm{m}$ which can swim with velocities more than a few $100 \mu \mathrm{m} / \mathrm{s}$ comparable to capillary flow velocity [1]. A number of microscale propulsion mechanisms have been reported such as external-fields-powered propulsions [2-5], microorganism-powered propulsions [6], and catalytic self-propulsions [7-10] by bubbles and/or phoretic flows. However, no mechanism has yet been established to meet the above requirements.

Considering those requirements, we have proposed the concept of the self-propelled microswimmer using electroosmotic propulsion (EOP) and biofuel cell (BFC) [11]. 
The BFC mechanism generates an open-circuit potential (OCP) by decomposing biofuels that are present in the human body, such as glucose and oxygen. The EOP mechanism creates self-propulsion velocity by a reaction force of electroosmotic flow (EOF), with the OCP as the energy source. According to the derived theoretical EOP model, its self-propulsion velocity is inversely proportional to the length of the microswimmer, while it is proportional to the OCP which does not depend on its size. Therefore, under conditions where both BFC and EOP mechanisms work, it can be expected that the smaller its microswimmer size, the faster its self-propulsion velocity. This remarkable feature can make this concept the best solution to those requirements. We proved the concept by observing the self-propulsions of $100 \mu \mathrm{m}$ prototypes with several $10 \mu \mathrm{m} / \mathrm{s}$ velocity in a glucose solution. The prototype consisted of one insulating layer made of UV-curable polymer SU-8 and two conductive polymer composite layers consisting of SU-8 and silver (Ag) nanoparticles (NPs) on which specific enzymes were immobilized and was fabricated by the optical photolithography using several photomasks and alignment steps. However, the standard photolithography was not suitable for further miniaturization of prototypes due to its insufficient resolution.

In this paper, we report new smaller prototypes capable of swimming with faster velocities in a glucose solution. We adopted the fabrication method suitable for them using femtosecond-laser 3D microlithography [12] and multi-materials composing of the conductive polymer composites (CPCs) and nonconductive polymer composite (NCPC). Then we evaluated the self-propulsion velocity of the prototypes experimentally to prove its validity of the smaller and faster feature.

\section{Materials and methods}

\section{Concept and physical model}

Figure 1a shows the concept. The BFC comprises a redoxelectrode pair, with through-holes for drainage. One electrode works as a bioanode oxidizing glucose, and the other as a biocathode reducing oxygen. The BFC mechanism generates an OCP between the electrodes by the redox reactions. The EOP mechanism comprises an electrically insulating tube arranged between them. Electric force by the OCP generates a plug flow of electrolyte within the tube (i.e., EOF). Its reaction force then drives the tube in the opposite direction to the EOF. Due to the EOF characteristics, this EOP mechanism can work effectively in microscale fluids.

The drag force acting on the outer surface of the tube is negligibly smaller than the reaction force inside it as proved in [11]. That condition sufficiently stands at the swimmer size more than $1 \mu \mathrm{m}$ assumed in this research.
Therefore, momentum conservation between the tube (with the mass of $m$ and the velocity of $v_{e o}$ ) and the inner bulk electrolyte (with the mass of $m_{f}$ and the velocity of $u_{e o}$ ) stands as follows:

$$
m v_{e o}+m_{f} u_{e o}=0 .
$$

Their relative velocity is equal to the so-called EOF velocity as follows:

$$
u_{e o}-v_{e o}=\frac{\epsilon \zeta}{\eta} \frac{\Delta \phi_{c a}}{L}
$$

where $L$ is the body length and $\Delta \phi_{c a}$ is the OCP generated by the BFC (Fig. 1b). $\zeta$ is the charged potential of the inner surface of the insulating tube (so-called "zeta potential"). $\epsilon$ and $\eta$ are the permittivity and viscosity of the electrolytic solution.

Self-propulsion velocity model $v_{e o}$ of the tube-shaped swimmer can be derived from Eqs. (1) and (2) as follows: [11]

$$
\begin{aligned}
v_{e o} & =-\frac{1}{1+m / m_{f}} \frac{\epsilon \zeta}{\eta} \frac{\Delta \phi_{c a}}{L}, \\
\frac{m}{m_{f}} & \simeq \frac{\rho_{t}}{\rho_{f}} \cdot\left(\left(\frac{D}{D_{t}}\right)^{2}-1\right) \quad\left(L_{e} \ll L\right),
\end{aligned}
$$

where $\rho_{t}$ and $\rho_{f}$ are the mass densities of the insulating tube and the electrolyte, respectively, and the total mass of the electrodes is neglected. The diameter ratio $D / D_{t}$ and the mass ratio $m / m_{f}$ are assumed to be constant. The OCP $\Delta \phi_{c a}$ does not depend on the electrode size. From Eq. (3), faster $v_{e o}$ is clearly expected by smaller body length $L$. Therefore, the proposed propulsion mechanism is theoretically suitable and advantageous for use as the self-propulsive microrobot.

\section{$10 \mu \mathrm{m}$ prototype design}

The configuration of the new prototype with a size of about $10 \mu \mathrm{m}$ is shown in Fig. 2a. The two electrodes are made from $\mathrm{CPCs}$ incorporating $\mathrm{SU}-8$, conductive NPs such as silver $(\mathrm{Ag})$ or gold $(\mathrm{Au})$, and anodic or cathodic enzymes with immobilized on the NPs. Other researchers have shown that the SU-8 composite has good electrical conductivity when the volume fraction of the metal NPs for SU-8 solid exceeds about 6 vol\% [13]. We used CPC incorporating the enzyme-immobilized AgNPs of 7 vol\%. The AgNPs had a mean particle size of $30 \mathrm{~nm}$. An anodic enzyme, such as glucose oxidase (GOx) or glucose dehydrogenase (GDH), are used for one electrode as a bioanode, a cathodic enzyme, such as laccase (LAC) or bilirubin oxidase (BOD), for another electrode as a biocathode. GOx was used as the anodic enzyme and LAC as the cathodic enzyme. 


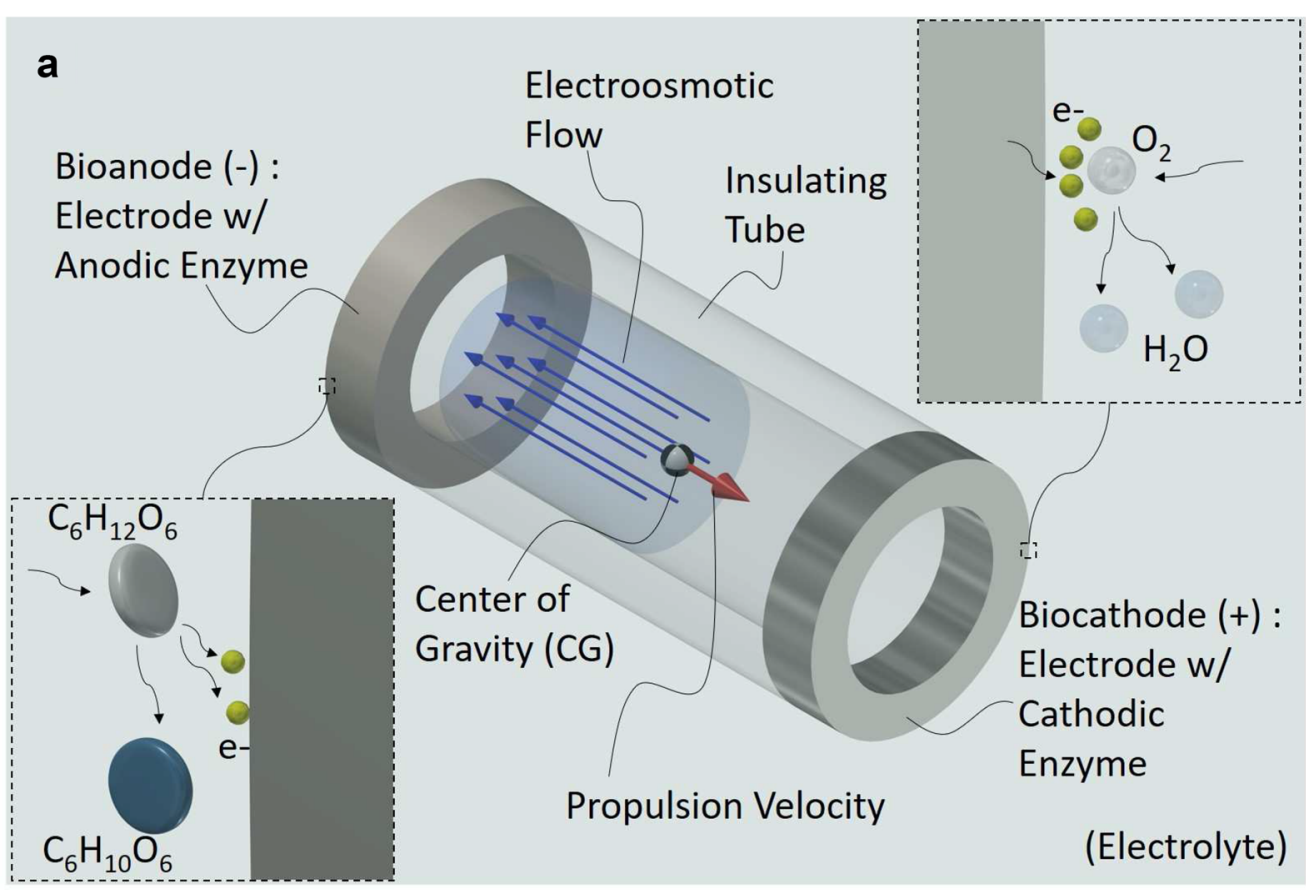

b

$\Delta \phi_{c a}:$ Open-Circuit Potential (OCP) Generated by Biofuel Cell (BFC)

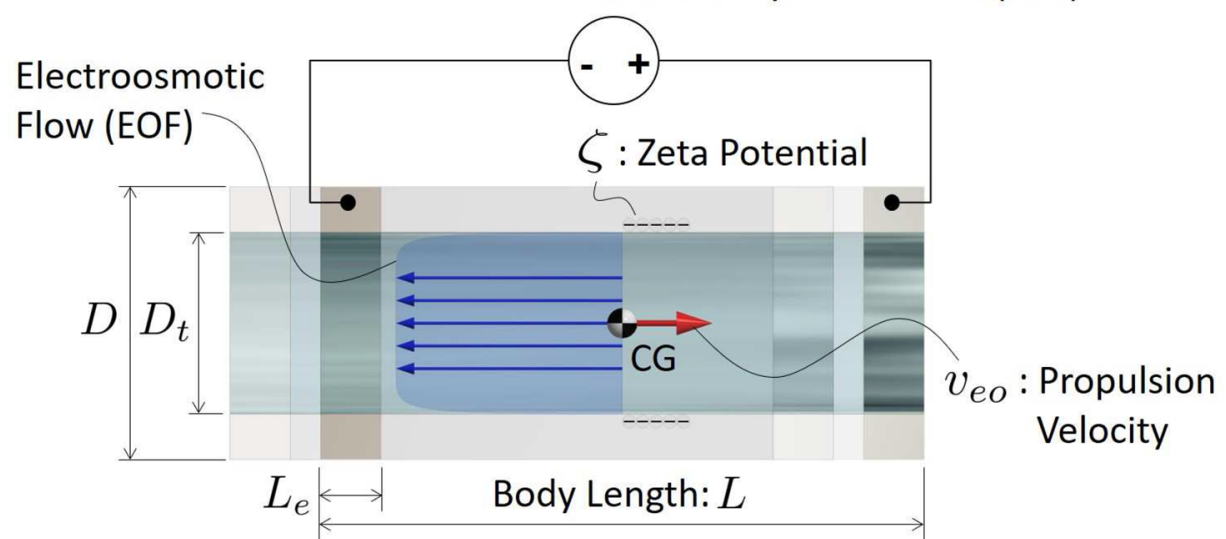

Fig. 1 Concept of microswimmer using BFC and EOP [1 1]. a Configuration and mechanisms. b Main parameters

To immobilize the enzymes to AgNPs, we adopted the covalent bonding method using self-assembled monolayers [14] of alkanethiols formed over the NP surfaces like our previous research [11]. The insulating tube is made from NCPC incorporating SU-8 and nonconductive NPs such as fullerene $\left(\mathrm{C}_{60}\right)$ or magnetite $\left(\mathrm{Fe}_{3} \mathrm{O}_{4}\right)$. As a result of several fabrication trials, NCPC was required as the insulating material instead of only SU-8 to use the same power condition of the femtosecond laser for the three layers. The optimal laser power for polymer composites with NPs becomes smaller than that for only polymers because of the radiation absorption and scattering by the NPs and their agglomerates [15]. We used the NCPC incorporating the fullerene $\left(\mathrm{C}_{60}\right)$ NPs of 2 vol\%. $\mathrm{C}_{60}$ NPs were suitable for NCPC because they have good dispersibility in uncured SU-8 fluid.

\section{Fabrication process}

The total fabrication process is shown in Fig. 2b. The fabrication process of $10 \mu \mathrm{m}$ prototypes used femtosecond laser 3D microlithography [12] by "Nanoscribe Photonic Professional" (63×, NA0.75). First, a dextran film as a water-soluble sacrificial layer was patterned on 

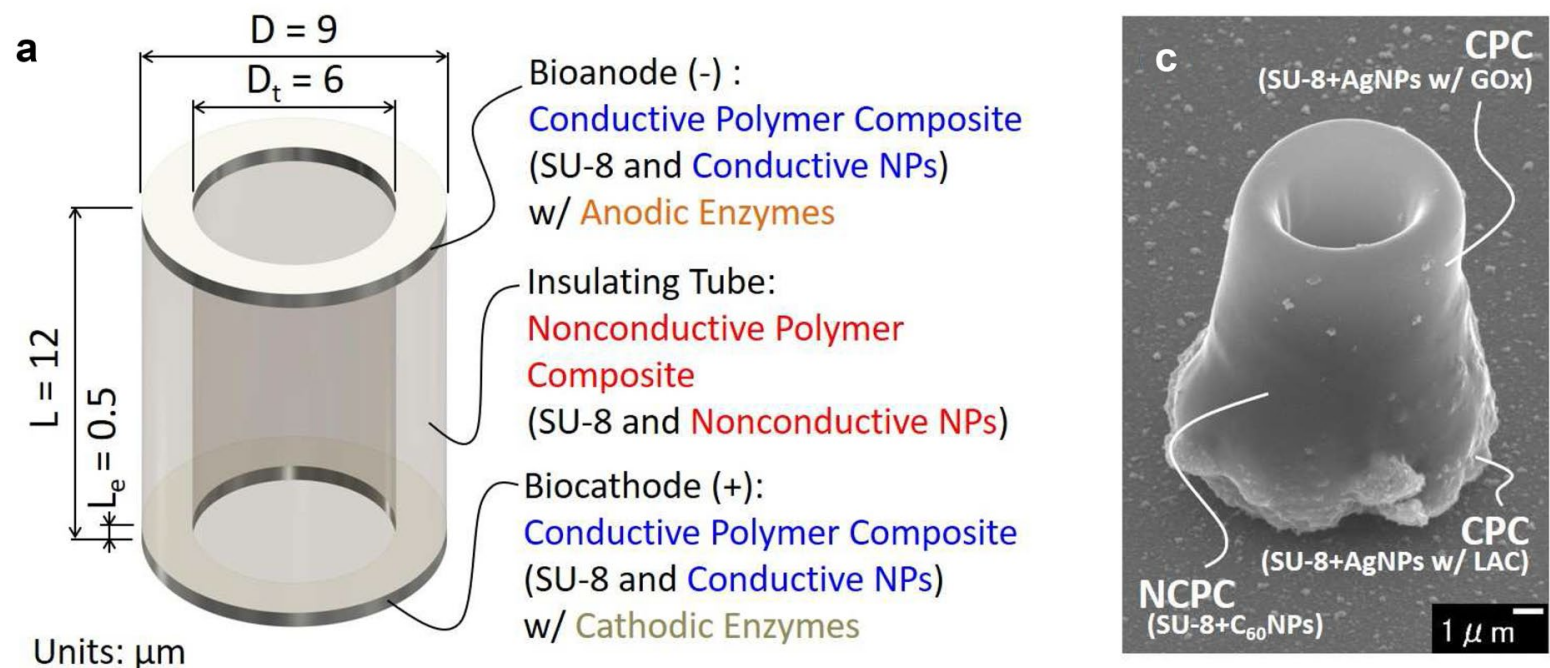

b

(b1) Sacrificial Layer Patterning (b2) All Layers Coating

(b3) 3D Two-Photon Polymerization
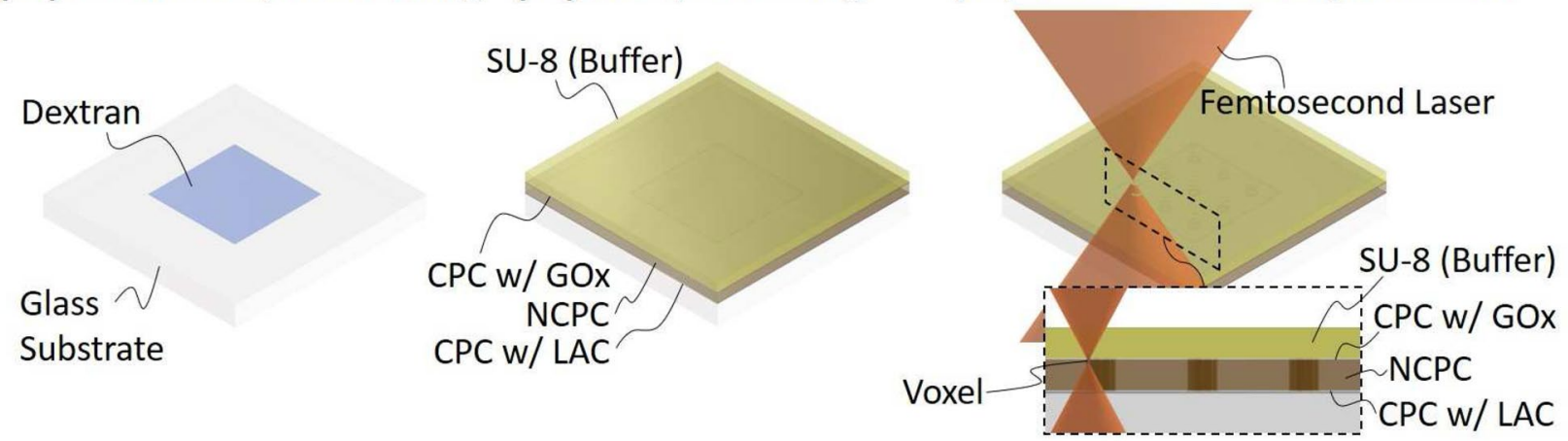

(b4) All Layers Development

(b5) Well Structure Bonding

(b6) Dextran Dissolution
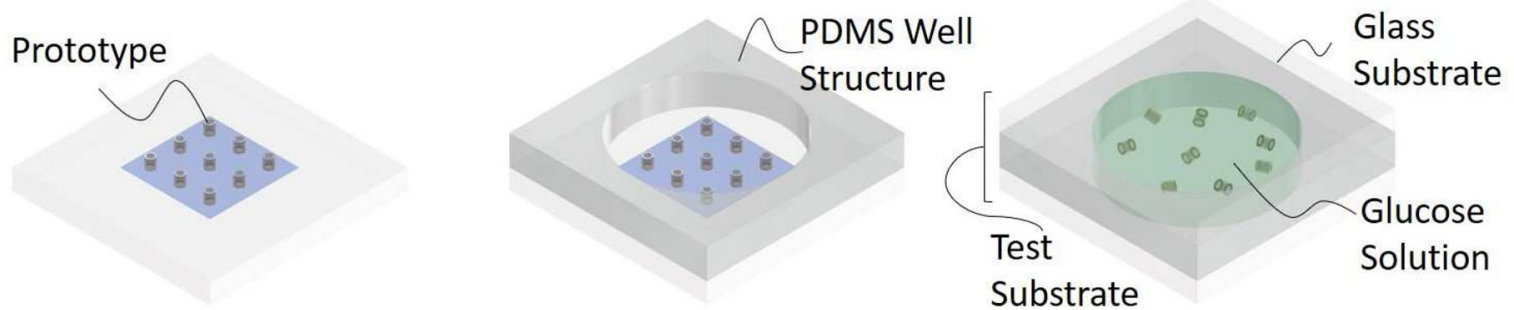

Fig. $210 \mu \mathrm{m}$ prototype. a Design and configuration. b Fabrication process. c Scanning electron microscopic (SEM) images of a fabricated prototype

a glass substrate by the lift-off method [16] as shown in Fig. 2b1. Next, three structural layers (CPC/NCPC/CPC) and an SU-8 buffer layer were coated on the substrate as shown in Fig. 2b2. The buffer layer was required for successful fabrication as described in detail later. To control the thickness of each layer, the viscosity of the material of each layer was adjusted in advance by diluting with cyclopentanone, which is a solvent of uncured SU-8 fluid. A focused spot (so-called "voxel") of the femtosecond laser (with the wavelength of $780 \mathrm{~nm}$ ) was scanned in tubelike regions of the structural layers as shown in Fig. $2 \mathrm{~b} 3$. Only those regions were polymerized due to two-photon absorption.

The optimization trials of fabrication conditions are summarized to Table 1. The optimal laser power was more than $3.0 \mathrm{~mW}$ for only SU-8 (Table 1(1)) while it was less than $2.5 \mathrm{~mW}$ for only CPC (Table $1(2)$ ). No optimal condition existed between $2.5-6.0 \mathrm{~mW}$ for the same 
Table 1 The optimization trials of fabrication conditions by using "Nanoscribe Photonic Professional" (63 x, NA0.75)

\begin{tabular}{|c|c|c|c|c|}
\hline Condition & Total thickness $(\mu \mathrm{m})$ & Laser power (mW) & Scan speed $(\mu \mathrm{m} / \mathrm{s})$ & Result \\
\hline (1) $1 \mathrm{~L}(\mathrm{SU}-8)$ & $0.5-9.0$ & $\geq 3.0$ & $10-30$ & OK \\
\hline (2) $1 \mathrm{~L}(\mathrm{CPC})$ & $0.5-1.0$ & $\leq 2.5$ & 10 & OK \\
\hline (3) $3 \mathrm{~L}(\mathrm{CPC} / \mathrm{SU}-8 / \mathrm{CPC})$ & 12.0 & $2.5-6.0$ & 10 & NG \\
\hline (4) $3 \mathrm{~L}(\mathrm{CPC} / \mathrm{NCPC} / \mathrm{CPC})$ & 12.0 & $\leq 2.5$ & 10 & NG \\
\hline (5) $3 \mathrm{~L}(\mathrm{CPC} / \mathrm{NCPC} / \mathrm{CPC})+$ buffer(SU-8) & $12.0+9.0$ (buffer) & $\leq 2.5$ & 10 & OK \\
\hline
\end{tabular}

L: Layer, CPC: SU-8 and AgNPs of 7 vol\%, NCPC: SU-8 and C60 NPs of 2 vol\%

three layers as the previous $100 \mu \mathrm{m}$ prototype configuration (CPC/SU-8/CPC) (Table 1(3)). The optimal laser power for polymer composites with NPs is assumed to be significantly different from that for only polymers because of the radiation absorption and scattering by the NPs and their agglomerates [15]. Therefore, the same laser power condition is expected for the three structural layers by using NCPC as the material of the insulating layer. However, without the top SU-8 buffer layer, the only lower electrode layer remained (Table 1(4)). The buffer was assumed to be required for the smoothnesses of both optical index and mechanical stress right above the top of the structural three layers. The SU-8 buffer is not polymerized because the laser power $2.5 \mathrm{~mW}$ is too small for it. From the above trials, the only successful condition Table 1(5) was derived.

The laser power was set to $2.5 \mathrm{~mW}$ and the scan speed $10 \mu \mathrm{m} / \mathrm{s}$. Finally, through chemical development and cleaning (Fig. 2b4), $10 \mu \mathrm{m}$ prototypes were successfully fabricated on the substrate as shown in Fig. 2c. During all baking processes, a baking temperature was set to less than $65^{\circ} \mathrm{C}$ to avoid deactivation of the enzymes [17]. The thickness of the CPC layer was about $0.5-1.0 \mu \mathrm{m}$. The length of the prototype was about 11.0-20.0 $\mu \mathrm{m}$ depending on the thickness of the NCPC layer.

A test substrate for experiments was fabricated by $\mathrm{O}_{2}$ plasma bonding of a well structure made of polydimethylpolysiloxane (PDMS) and the glass substrate with the prototypes (Fig. 2b5). A glucose solution (67 $\mathrm{mM} \beta$-D-glucose, $150 \mathrm{mM} \mathrm{NaCl}$, and fluorescent polystyrene (PS) beads with the size of $3 \mu \mathrm{m}$ in $10 \mathrm{mM}, 7.4 \mathrm{pH}$ phosphate-buffered saline (PBS)) was added in the well. Its glucose concentration was set close to that of the blood of a diabetic patient [1] as the maximum in vivo value. That is the same condition as the previous experiments [11]. The dextran film is water-soluble, so the prototypes were released from the substrate immediately when the solution was added (Fig. 2b6).

\section{Experimental methods}

We carried out experiments observing self-propulsions of $10 \mu \mathrm{m}$ prototypes in a glucose solution using an inverted microscope as shown in Fig. 3. We acquired the videos of the movements from microscopic images, tracked prototypes, and identified propulsion velocities. Finally, we compared those results with the theoretical curves and the results of the previous $100 \mu \mathrm{m}$ prototypes.

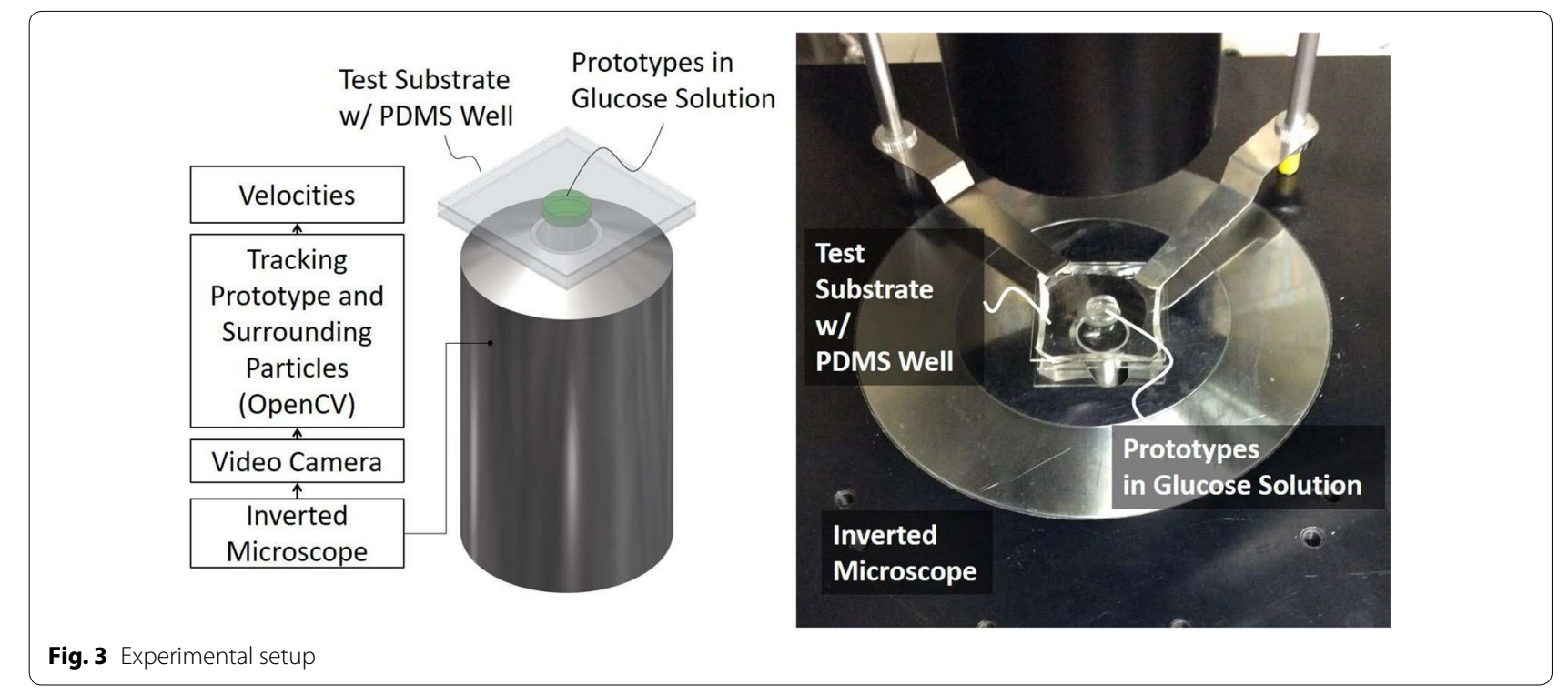




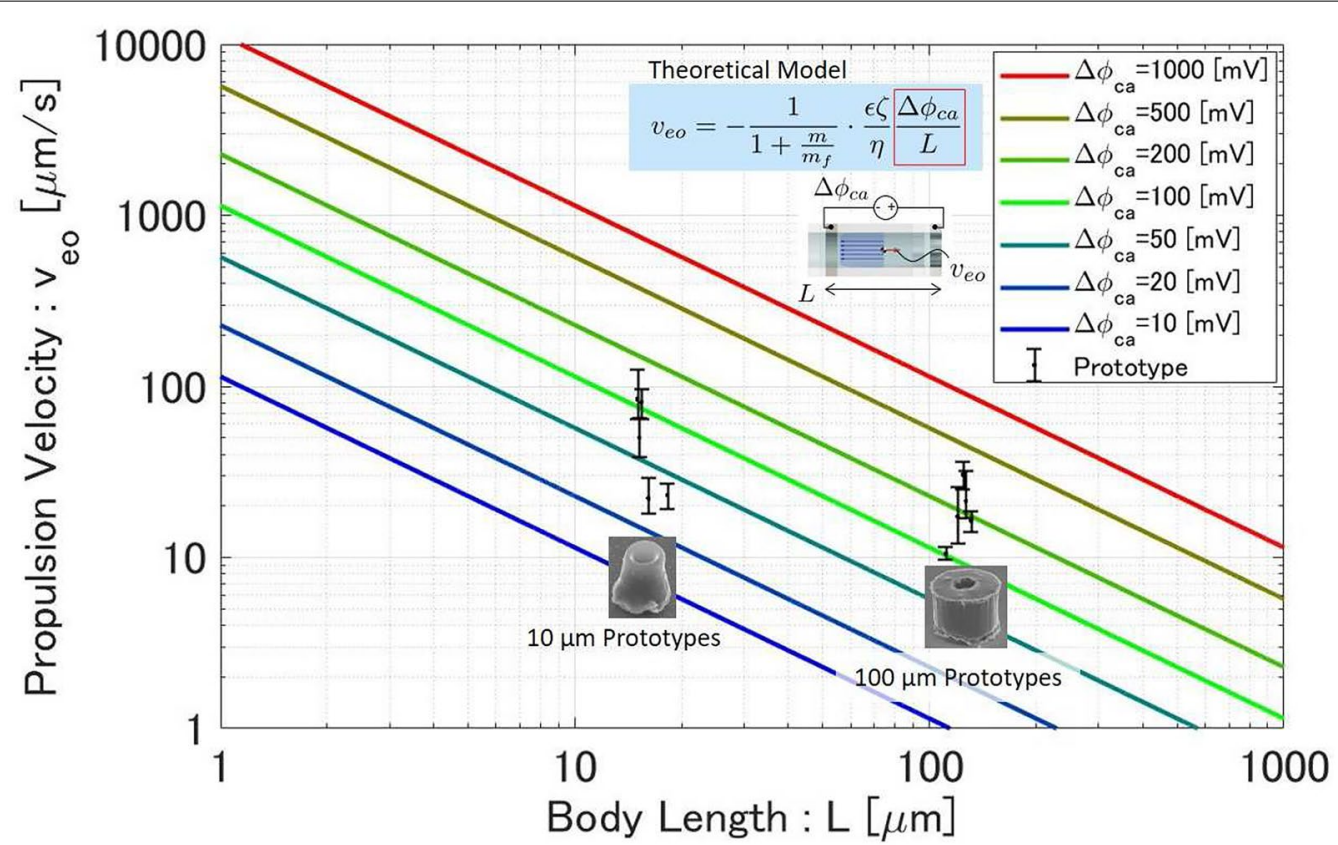

Fig. 4 Propulsion velocity against body length: theory and experiments. A point denotes a mean value of $v_{e o}$ experimentally identified and a bar denotes the range. The experimental data of $100 \mu \mathrm{m}$ prototypes were referenced from [1 1]

The test substrate was covered with a glass substrate and set on a stage just above the microscope. We can observe and identify unnecessary convective flows by tracking the PS beads in the glucose solution.

After acquiring the video from the microscope, a red circle and a white curve were added to that using "OpenCV" to track the center of gravity (CG) of the prototype and its trajectory. Blue circles with white curves were also added to that by optical-flow processing and they were trajectories of the PS beads or other residues in the surrounding fluid and indicated surrounding convective flows.

The velocities $v_{p}, v_{f l o w}$, and $v_{e o}$ against time $t$ were extracted from the image-processed video with the sampling interval of $0.1 \mathrm{~s}$, where $v_{p}$ is the velocity of the CG of the prototype, $v_{\text {flow }}$ is the mean velocity of the surrounding convective flows in the same direction, and $v_{p}-v_{\text {flow }}$ is identified as the self-propulsion velocity $v_{e o}$.

\section{Results and discussion}

\section{Theoretical performance}

Theoretical performance curves were shown in Fig. 4 which were calculated using Eq. (3) with the parameter values from Table 2 and Fig. 1b. From the theoretical curves, faster $v_{e o}$ is clearly expected by smaller body length $L$. If $L$ can be made smaller than $10 \mu \mathrm{m}, v_{e o}$ is expected to exceed $1 \mathrm{~mm} / \mathrm{s}$, which is close to the blood

Table 2 Parameters [11]

\begin{tabular}{lllll}
\hline Meaning & Part & Symbol & Value (units) & Comment \\
\hline Permittivity & Electrolyte & $\epsilon$ & $6.580 \times 10^{-10}(\mathrm{~F} / \mathrm{m})$ & Water (310 K) \\
Viscosity & Electrolyte & $\eta$ & $0.692 \times 10^{-3}(\mathrm{~Pa} \mathrm{~s})$ & Water (310 K) \\
Mass density & Electrolyte & $\rho_{f}$ & $993\left(\mathrm{~kg} / \mathrm{m}^{3}\right)$ & Water (310 K) \\
Mass density & Insulating tube & $\rho_{t}$ & $1190\left(\mathrm{~kg} / \mathrm{m}^{3}\right)$ & SU-8 [18] \\
Zeta potential & Insulating tube & $\zeta$ & $-0.030(\mathrm{~V})$ & SU-8 [19] \\
OCP & Electrodes & $\Delta \phi_{c a}$ & $<1.18(\mathrm{~V})$ & Theoretical supremum \\
& & & from the Gibbs free \\
Diameter ratio & & $D / D_{t}$ & 1.50 & energy [20]
\end{tabular}



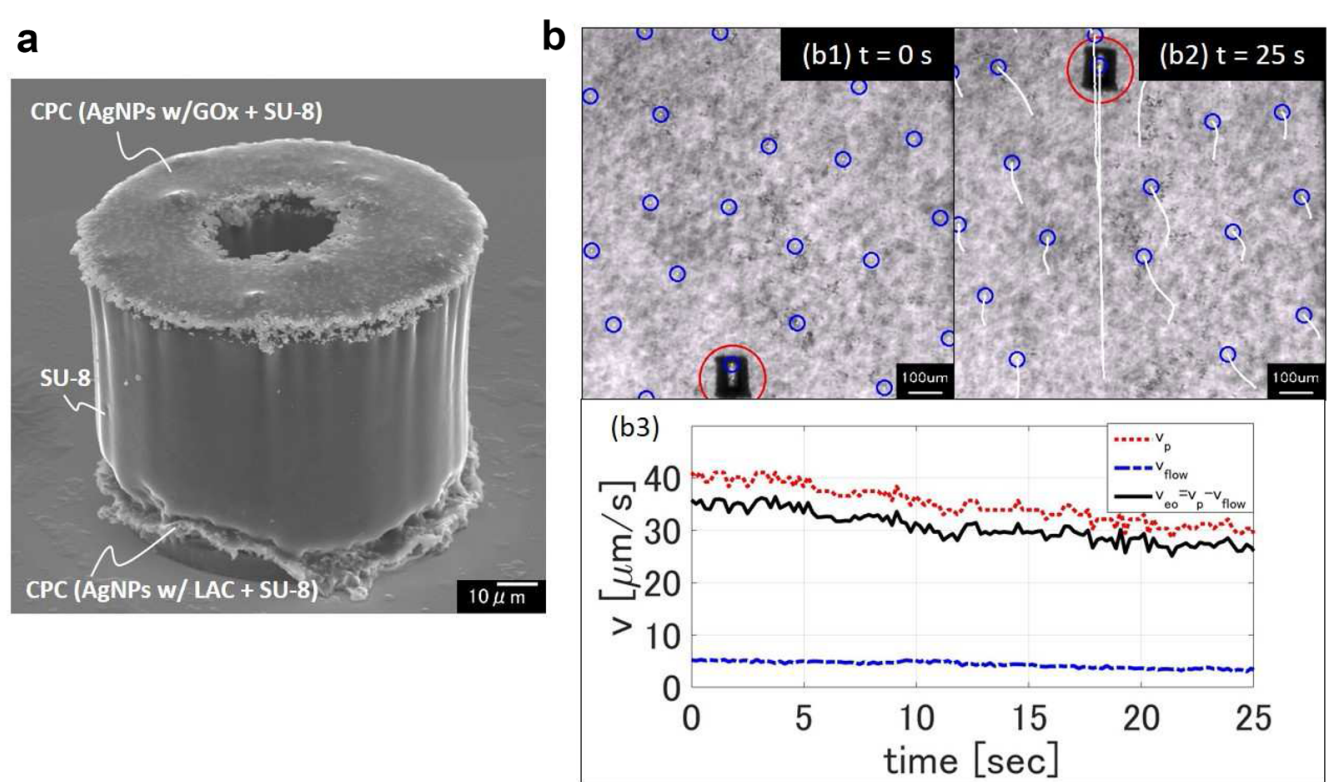

Fig. 5 Results of $100 \mu \mathrm{m}$ prototypes [11]. a SEM image of the $100 \mu \mathrm{m}$ prototype fabricated by the standard photolithography. b Self-propulsion of the fastest $100 \mu \mathrm{m}$ prototype in a glucose solution. (b1), (b2) Snapshots extracted from a video. A red circle, blue circles, and white curves added by "OpenCV" represent the center of gravity (CG) of the prototype, surrounding particles indicating convective flows, and their trajectories, respectively. (b3) Velocities against time $t$ extracted from the video with the sampling interval of $0.1 \mathrm{~s}$, where $v_{p}$ is the velocity of the CG of the prototype, $v_{f l o w}$ the mean velocity of the surrounding convective flows in the same direction, and $v_{p}-v_{\text {flow }}$ the identified self-propulsion velocity $v_{e o}$
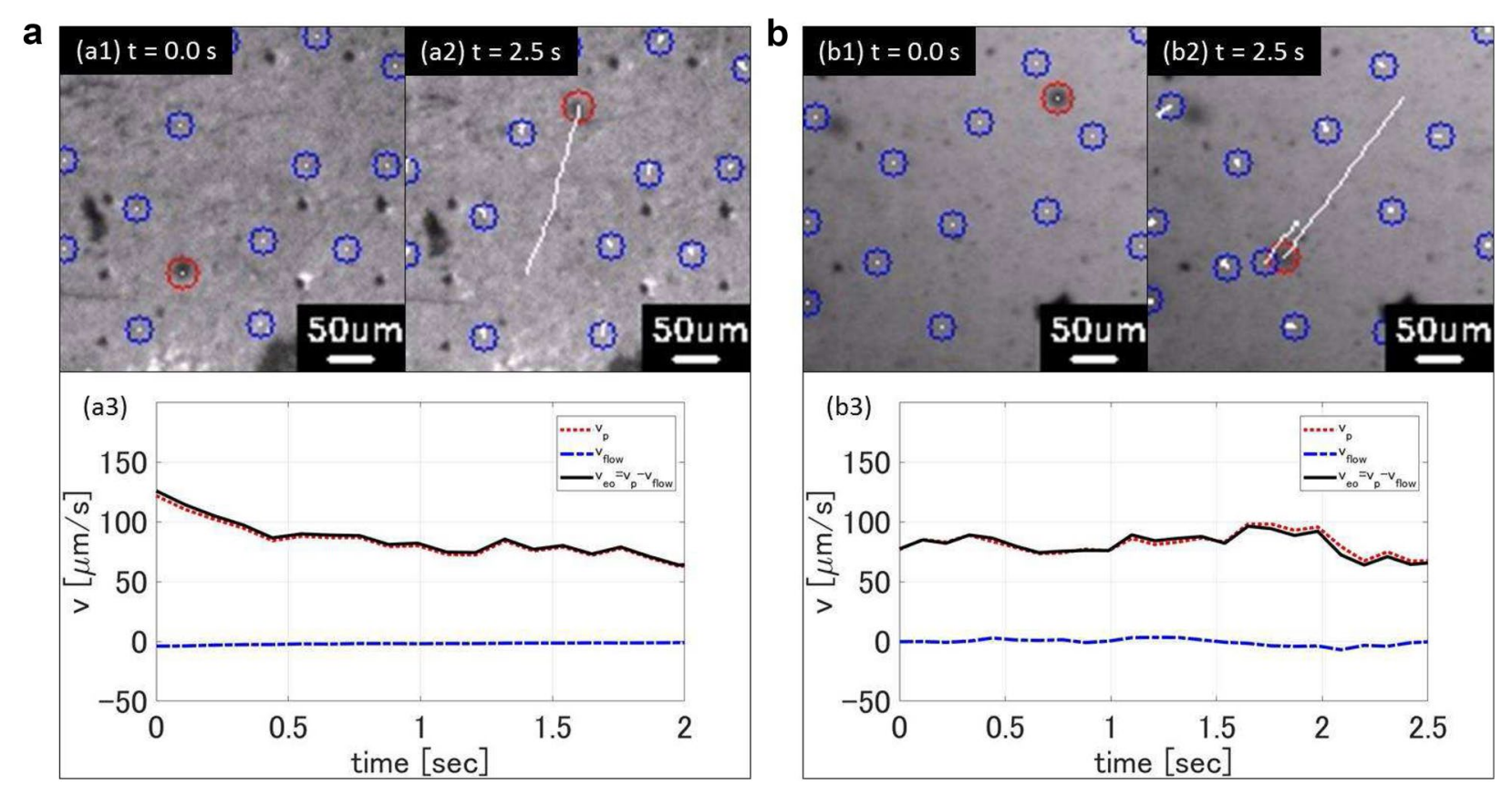

Fig. 6 Self-propulsions of the two fastest $10 \mu \mathrm{m}$ prototypes in a glucose solution. a The fastest prototype. $\mathbf{b}$ Another prototype. These were processed in the same way as Fig. 5b. Additional files 1 and 2 are the movies of experiments corresponding to $\mathbf{a}$ and $\mathbf{b}$, respectively. 
flow in the human capillary system [1]. However, this depends on $\Delta \phi_{c a}, \zeta$, and $\eta$.

\section{Self-propulsion of previous $100 \mu \mathrm{m}$ prototypes}

In the previous study, we fabricated $100 \mu \mathrm{m}$ prototypes by the standard photolithography with several alignment and exposure steps as shown in Fig. 5a, and the selfpropulsions of them were observed in a glucose solution [11]. Figure $5 \mathrm{~b}$ shows the fastest results. The trajectory of the CG shows that the movement was in a straight line. Over $25 \mathrm{~s}$, the prototype moved $848 \mu \mathrm{m}$. its maximum velocity was identified as $35 \mu \mathrm{m} / \mathrm{s}$ by removing the surrounding convective flow velocities as shown in Fig. $5 \mathrm{~b} 3$.

\section{Self-propulsion of $10 \mu \mathrm{m}$ prototypes}

Figure 6 shows self-propulsion results of the two fastest $10 \mu \mathrm{m}$ prototypes. Figure $6 \mathrm{a} 1, \mathrm{a} 2$ show snapshots extracted from the image-processed video. Figure $6 \mathrm{a} 3$ shows the velocities $v_{p}, v_{\text {flow }}$, and $v_{e o}$ against time $t$ extracted from the video. Figure $6 \mathrm{~b}$ shows another result. The self-propulsions of the $10 \mu \mathrm{m}$ prototypes were observed (Fig. 6a2, b2). Their trajectories (the white curves with the red circles) show that they moved in the straight lines. There were almost no convective flows (the white curves with the blue circles) around them. Over 2.5 $\mathrm{s}$, the fastest prototype moved $215 \mu \mathrm{m}$ (Fig. 6a2). A maximum velocity of the prototype was identified as $126 \mu \mathrm{m} / \mathrm{s}$ from Fig. 6a3. A maximum velocity of the fastest 100 $\mu \mathrm{m}$ prototype was $35 \mu \mathrm{m} / \mathrm{s}$ [11]. From the experimental results, the faster velocity by the smaller body size was confirmed as theoretically expected.

\section{Miniaturization effect}

Finally, we confirmed the validity of the experimentally identified velocities $v_{e o}$ of the five $10 \mu \mathrm{m}$ prototypes by comparing with the theory and those of $100 \mu \mathrm{m}$ prototypes [11] (Fig. 4).

The mean of $v_{e o}$ in the $10 \mu \mathrm{m}$ prototypes was $100 \mu \mathrm{m} / \mathrm{s}$ and was better than that in the $100 \mu \mathrm{m}$ prototypes, which was $19 \mu \mathrm{m} / \mathrm{s}$. However, the range of $\nu_{e o}$ in the $10 \mu \mathrm{m}$ prototypes was $18-126 \mu \mathrm{m} / \mathrm{s}$, while that in the $100 \mu \mathrm{m}$ prototypes was $10-35 \mu \mathrm{m} / \mathrm{s}$. The reasons for the large variation in $10 \mu \mathrm{m}$ prototypes are not exactly clear, but the variation might be due to the diameter ratio $D / D_{t}$ and the OCP $\Delta \phi_{c a}$ of the prototypes from Eq. (3).

We assume a dimensional error $d D=1.00 \mu \mathrm{m}$ and $(D+d D) /\left(D_{t}-d D\right)=(9.00+1.00) /(6.00-1.00)=2.00$ instead of the nominal value $D / D_{t}=1.50$. Theoretical $v_{e o}$ with $d D$ is calculated as $54.4 \%$ of its nominal value using Eq. (3). The sensitivity of the $D / D_{t}$ variation of $10 \mu \mathrm{m}$ prototypes is relatively large because of its smaller $D$.
Also the OCP $\Delta \phi_{c a}$ is highly dependent on the activities of the enzymes. Using Eq. (3) with the parameter values from Table 2, the OCPs $\Delta \phi_{c a}$ of the experimental $v_{e o}$ were identified as less than $200 \mathrm{mV}$ and are worse than those of $100 \mu \mathrm{m}$ prototypes identified as less than 400 $\mathrm{mV}$ [11]. The focused spot of femtosecond-laser might have resulted in more inactivation of the enzymes than UV exposure of the standard photolithography.

We consider that resolving this variation issue is one important future work.

\section{Conclusions}

We adopted the fabrication method using femtosecondlaser 3D microlithography and multi-materials composing of the conductive polymer composites (CPCs) and nonconductive polymer composite (NCPC) and succeeded in fabricating $10 \mu \mathrm{m}$ prototypes. Then we demonstrated more than $100 \mu \mathrm{m} / \mathrm{s}$ velocity of the prototype experimentally and proved its validity of the smaller and faster feature. From this experimental verification, this concept is suitable for future biomedical microrobots.

Many challenges still remain for medical applications, such as improving yields, enhancing performance stability, ensuring biocompatibility, and so on. In the next steps, we will develop its strategy to control the microswimmers as microrobots. Also, another embodiments using BFC and EOF mechanisms are biofuel-driven wireless micropumps. They are expected to be applied to perfusions of biological fluids at small local sites inside or outside the human body.

\section{Supplementary information}

Supplementary information accompanies this paper at https://doi. org/10.1186/s40648-019-0146-X.

Additional file 1. Movie of experiment corresponding to Fig. 6a. Additional file 2. Movie of experiment corresponding to Fig. 6b.

\section{Abbreviations}

BFC: Biofuel cell; EO: Electroosmotic; EOF: Electroosmotic flow; EOP: Electroosmotic propulsion; OCP: Open-circuit potential; CPC: Conductive polymer composite; NCPC: Nonconductive polymer composite; NP: Nanoparticle; Ag: Silver; $\mathrm{C}_{60}$ : fullerene; $\mathrm{GOx}$ : Glucose oxidase; LAC: Laccase.

\section{Authors' contributions}

TY and FA desinged research. TY performed research. TY analyzed the data. TY and FA wrote the paper. FA provided critical advice on the research strategy and design. Both authors read and approved the final manuscript.

\section{Funding}

This work was supported by "Japan Society for the Promotion of Science" Grants-in-Aid for Scientific Research (KAKENHI) (Grant JP19K21943).

\section{Availability of data and materials}

Not applicable.

Ethics approval and consent to participate Not applicable. 


\section{Consent for publication}

Not applicable.

\section{Competing interests}

The authors declare that they have no competing interests.

Received: 21 November 2019 Accepted: 4 December 2019

Published online: 19 December 2019

\section{References}

1. Freitas RA Jr (1999) Nanomedicine, vol 1. Basic capabilities. Landes Bioscience, Georgetown

2. Chang ST, Paunov VN, Petsev DN, Velev OD (2007) Remotely powered self-propelling particles and micropumps based on miniature diodes. Nat Mater 6(3):235-240

3. Bell DJ, Leutenegger S, Hammar KM, Dong LX, Nelson BJ (2007) Flagellalike propulsion for microrobots using a nanocoil and a rotating electromagnetic field. In: Proceedings of IEEE international conference robotics automation. IEEE, pp. 1128-1133

4. Ghosh A, Fischer P (2009) Controlled propulsion of artificial magnetic nanostructured propellers. Nano Lett 9(6):2243-2245

5. Wu Z, Si T, Gao W, Lin X, Wang J, He Q (2016) Superfast near-infrared lightdriven polymer multilayer rockets. Small 12(5):577-582

6. Martel S, Tremblay CC, Ngakeng S, Langlois G (2006) Controlled manipulation and actuation of micro-objects with magnetotactic bacteria. Appl Phys Lett 89(23):233904

7. Sanchez S, Ananth AN, Fomin VM, Viehrig M, Schmidt OG (2011) Superfast motion of catalytic microjet engines at physiological temperature. J Am Chem Soc 133(38):14860-14863

8. Paxton WF, Sen A, Mallouk TE (2005) Motility of catalytic nanoparticles through self-generated forces. Chem Eur J 11(22):6462-6470

9. Mano N, Heller A (2005) Bioelectrochemical propulsion. J Am Chem Soc 127(33):11574-11575

10. Ma X, Jannasch A, Albrecht UR, Hahn K, Miguel-López A, Schäffer E, Sánchez S (2015) Enzyme-powered hollow mesoporous janus nanomotors. Nano Lett 15(10):7043-7050
11. Yamanaka T, Arai F (2018) Self-propelled swimming microrobot using electroosmotic propulsion and biofuel cell. IEEE Robot Autom Lett 3(3):1787-1792

12. Maruo S, Nakamura O, Kawata S (1997) Three-dimensional microfabrication with two-photon-absorbed photopolymerization. Opt Lett 22(2):132-134

13. Jiquet S, Bertsch A, Hofmann H, Renaud P (2005) Conductive SU8 photoresist for microfabrication. Adv Funct Mater 15(9):1511-1516

14. Patel N, Davies MC, Hartshorne M, Heaton RJ, Roberts CJ, Tendler SJ, Williams PM (1997) Immobilization of protein molecules onto homogeneous and mixed carboxylate-terminated self-assembled monolayers. Langmuir 13(24):6485-6490

15. Suter M, Zhang L, Siringil EC, Peters C, Luehmann T, Ergeneman O, Peyer KE, Nelson BJ, Hierold C (2013) Superparamagnetic microrobots: fabrication by two-photon polymerization and biocompatibility. Biomed Microdev 15(6):997-1003

16. Nagai M, Tanizaki K, Kawashima T, Shibata T (2014) Direct assembly of movable parts in a microchannel using a water-soluble sacrificial layer for biohybrid systems. In: 2014 international symposium on micro-nanomechatronics and human science (MHS). IEEE, pp. 1-4

17. Psoma SD, van der Wal PD, Frey O, de Rooij NF, Turner AP (2010) A novel enzyme entrapment in SU-8 microfabricated films for glucose microbiosensors. Biosens Bioelect 26(4):1582-1587

18. Bao XQ, Bontemps A, Grondel S, Cattan E (2011) Design and fabrication of insect-inspired composite wings for mav application using mems technology. J Micromech Microeng 21(12):125020

19. Sikanen T, Tuomikoski S, Ketola RA, Kostiainen R, Franssila S, Kotiaho T (2005) Characterization of SU-8 for electrokinetic microfluidic applications. Lab Chip 5(8):888-896

20. Soukharev V, Mano N, Heller A (2004) A four-electron $\mathrm{O}_{2}$-electroreduction biocatalyst superior to platinum and a biofuel cell operating at $0.88 \mathrm{~V}$. J Am Chem Soc 126(27):8368-8369

\section{Publisher's Note}

Springer Nature remains neutral with regard to jurisdictional claims in published maps and institutional affiliations.

\section{Submit your manuscript to a SpringerOpen ${ }^{\circ}$ journal and benefit from:}

- Convenient online submission

- Rigorous peer review

- Open access: articles freely available online

- High visibility within the field

- Retaining the copyright to your article

Submit your next manuscript at $\boldsymbol{\nabla}$ springeropen.com 